\title{
Existence and Uniqueness of Solutions for Initial Value Problem of Nonlinear Fractional Differential Equations
}

\author{
Qiuping Li, ${ }^{1}$ Shurong Sun, ${ }^{1,2}$ Ping Zhao, ${ }^{3}$ and Zhenlai Han ${ }^{1}$ \\ ${ }^{1}$ School of Mathematical Sciences, University of Jinan, Shandong, Jinan 250022, China \\ ${ }^{2}$ Department of Mathematics and Statistics, Missouri University of Science and Technology, Rolla, \\ MO 65409-0020, USA \\ ${ }^{3}$ School of Control Science and Engineering, University of Jinan, Shandong, Jinan 250022, China \\ Correspondence should be addressed to Shurong Sun, sshrong@163.com
}

Received 8 May 2012; Revised 12 June 2012; Accepted 13 June 2012

Academic Editor: Dumitru Baleanu

Copyright (c) 2012 Qiuping Li et al. This is an open access article distributed under the Creative Commons Attribution License, which permits unrestricted use, distribution, and reproduction in any medium, provided the original work is properly cited.

We discuss the initial value problem for the nonlinear fractional differential equation $L(D) u=$ $f(t, u), t \in(0,1], u(0)=0$, where $L(D)=D^{s_{n}}-a_{n-1} D^{s_{n-1}}-\cdots-a_{1} D^{s_{1}}, 0<s_{1}<s_{2}<\cdots<s_{n}<1$, and $a_{j}<0, j=1,2, \ldots, n-1, D^{s_{j}}$ is the standard Riemann-Liouville fractional derivative and $f:[0,1] \times \mathbb{R} \rightarrow \mathbb{R}$ is a given continuous function. We extend the basic theory of differential equation, the method of upper and lower solutions, and monotone iterative technique to the initial value problem. Some existence and uniqueness results are established.

\section{Introduction}

Fractional calculus is a generalization of ordinary differentiation and integration to arbitrary noninteger order, so fractional differential equations have wider application. Fractional differential equations have gained considerable importance; it can describe many phenomena in various fields of science and engineering such as control, porous media, electrochemistry, viscoelasticity, and electromagnetic.

In the recent years, there has been a significant development in fractional calculus and fractional differential equations; see Kilbas et al. [1], Miller and Ross [2], Podlubny [3], Baleanu et al. [4], and so forth. Research on the solutions of fractional differential equations is very extensive, such as numerical solutions, see El-Mesiry et al. [5] and Hashim et al. [6], mild solutions, see Chang et al. [7] and Chen et al. [8], the existence and uniqueness of solutions for initial and boundary value problem, see [9-30], and so on. 
With the deep study, many papers that studied the fractional equations contained more than one fractional differential operator; see [16-20].

Babakhani and Daftardar-Gejji in [16] considered the initial value problem of nonlinear fractional differential equation

$$
L(D) u=f(t, u), \quad u(0)=0, \quad 0<t<1
$$

By using Banach fixed point theorem and fixed point theorem on a cone some results of existence and uniqueness of solutions are established.

Zhang in [17] studied the singular initial value problem for fractional differential equation by nonlinear alternative of Leray-Schauder theorem:

$$
L(D) u=f(t, u),\left.\quad t^{1-s_{n}} u(t)\right|_{t=0}=0, \quad 0<t \leq 1 .
$$

In above two equations, $L(D)$ is defined $L(D):=D^{s_{n}}-a_{n-1} D^{s_{n-1}}-\cdots-a_{1} D^{s_{1}}$, where $0<s_{1}<s_{2}<\cdots<s_{n}<1$, and $a_{j}>0, j=1,2, \ldots, n-1, D^{s_{j}}$ is the standard Riemann-Liouville fractional derivative.

McRae in [14] studied the initial value problem by the method of upper and lower solutions and monotone iterative technique:

$$
\begin{gathered}
D^{q} u=f(t, u), \quad t \in\left(t_{0}, T\right], 0<q<1, \\
u\left(t_{0}\right)=u^{0}=\left.u(t)\left(t-t_{0}\right)^{1-q}\right|_{t=t_{0}} .
\end{gathered}
$$

In this paper, we use similar method as in [16] to consider the initial value problem:

$$
\begin{gathered}
L(D) u=f(t, u), \quad t \in(0,1], \\
u(0)=0,
\end{gathered}
$$

where $L(D)=D^{s_{n}}-a_{n-1} D^{s_{n-1}}-\cdots-a_{1} D^{s_{1}}, 0<s_{1}<s_{2}<\cdots<s_{n}<1$, and $a_{j}<0, j=1,2, \ldots, n-1$, $D^{s_{j}}$ is the standard Riemann-Liouville fractional derivative and $f:[0,1] \times \mathbb{R} \rightarrow \mathbb{R}$ is a given continuous function. 
Since $f$ is assumed continuous, the IVP (1.4) is equivalent to the following Volterra fractional integral equation:

$$
u(t)=\sum_{j=1}^{n-1} \frac{a_{j}}{\Gamma\left(s_{n}-s_{j}\right)} \int_{0}^{t}(t-s)^{s_{n}-s_{n-1}-1} u(s) d s+\frac{1}{\Gamma\left(s_{n}\right)} \int_{0}^{t}(t-s)^{s_{n}-1} f(s, u(s)) d s .
$$

In Section 2, we give some definitions and lemmas that will be useful to our main results. In Section 3, we will use the basic theory of differential equation, the method of upper and lower solutions, and monotone iterative technique to investigate the initial value problem (1.4), and some existence and uniqueness results are established. In Section 4, an example is presented to illustrate the main results.

\section{Preliminaries}

In this section, we need the following definitions and lemmas that will be useful to our main results. These materials can be found in the recent literatures; see $[1,11,16]$.

Definition 2.1 (see [1]). Let $\Omega=[a, b](-\infty<a<b<+\infty)$ be a finite interval on the real axis $\mathbb{R}$. The Riemann-Liouville fractional integrals $I_{a+}^{\alpha} f$ and $I_{b-}^{\alpha} f$ of order $\alpha>0$ are defined by

$$
\begin{aligned}
& I_{a+}^{\alpha} f(x)=\frac{1}{\Gamma(\alpha)} \int_{a}^{x}(x-t)^{\alpha-1} f(t) d t, \quad x>a, \\
& I_{b-}^{\alpha} f(x)=\frac{1}{\Gamma(\alpha)} \int_{x}^{b}(x-t)^{\alpha-1} f(t) d t, \quad x<b,
\end{aligned}
$$

respectively. Here $\Gamma(\alpha)$ is the Gamma function. These integrals are called the left-sided and the right-sided fractional integrals. We denote $I_{0+}^{\alpha} f(x)$ by $I^{\alpha} f(x)$ in the following paper.

Definition 2.2 (see [1]). Let $\Omega=[a, b](-\infty<a<b<+\infty)$ be a finite interval on the real axis $\mathbb{R}$. The Riemann-Liouville fractional derivatives $D_{a+}^{\alpha} f$ and $D_{b-}^{\alpha} f$ of order $\alpha>0$ are defined by

$$
\begin{array}{cc}
D_{a+}^{\alpha} f(x)=\left(\frac{d}{d x}\right)^{n}\left(I_{a+}^{n-\alpha}\right) f(x)=\frac{1}{\Gamma(n-\alpha)}\left(\frac{d}{d x}\right)^{n} \int_{a}^{x}(x-t)^{n-\alpha-1} f(t) d t, & x>a, \\
D_{b-}^{\alpha} f(x)=\left(-\frac{d}{d x}\right)^{n}\left(I_{b-}^{n-\alpha}\right) f(x)=\frac{1}{\Gamma(n-\alpha)}\left(-\frac{d}{d x}\right)^{n} \int_{x}^{b}(x-t)^{n-\alpha-1} f(t) d t, & x<b,
\end{array}
$$

respectively, where $n=[\alpha]+1,[\alpha]$ means the integral part of $\alpha$. These derivatives are called the left-sided and the right-sided fractional derivatives. We denote $D_{0+}^{\alpha} f(x)$ by $D^{\alpha} f(x)$ in the following paper. 
Definition 2.3. Letting $v, w \in C([0,1], \mathbb{R})$ be locally Hölder continuous with exponent $s_{n}<$ $\lambda<1$, we say that $w$ is an upper solution of (1.4) if

$$
\begin{gathered}
L(D) w \geq f(t, w), \\
w(0) \geq 0,
\end{gathered}
$$

and $v$ is a lower solution of (1.4) if

$$
\begin{gathered}
L(D) v \leq f(t, v), \\
v(0) \leq 0 .
\end{gathered}
$$

Next, we will list the following lemma from [11] that is useful for our main results.

Lemma 2.4 (see [11, Lemma 2.1]). Let $m \in C([0,1], \mathbb{R})$ be locally Hölder continuous with exponent $q<\lambda<1$ such that for any $t_{1} \in(0,1]$, we have

$$
m\left(t_{1}\right)=0, \quad m(t) \leq 0 \quad \text { for } 0 \leq \mathrm{t} \leq \mathrm{t}_{1}
$$

Then it follows that $D^{q} m\left(t_{1}\right) \geq 0$.

Corollary 2.5. Let $m \in C([0,1], \mathbb{R})$ be locally Hölder continuous with exponent $s_{n}<\lambda<1$ such that for any $t_{1} \in(0,1]$, we have

$$
m\left(t_{1}\right)=0, \quad m(t) \leq 0 \quad \text { for } 0 \leq \mathrm{t} \leq \mathrm{t}_{1}
$$

Then it follows that $L(D) m\left(t_{1}\right) \geq 0$ provided $a_{j}<0, j=1,2, \ldots, n-1$.

Lemma 2.6. Let $\left\{u_{\epsilon}(t)\right\}$ be a family of continuous functions on $[0,1]$, for each $\epsilon>0$, where $L(D) u_{\epsilon}(t)=f\left(t, u_{\epsilon}(t)\right), u_{\epsilon}(0)=0$ and $\left|f\left(t, u_{\epsilon}(t)\right)\right| \leq M$ for $0 \leq t \leq 1$. Then the family $\left\{u_{\epsilon}(t)\right\}$ is equicontinuous on $[0,1]$.

Proof. Since $\left\{u_{\epsilon}(t)\right\}$ is a family of continuous functions on $[0,1]$, there exists $l>0$ such that $\left|u_{\epsilon}(t)\right| \leq l$ for $0 \leq t \leq 1$.

Let $\delta<\min \left\{\left(\sum_{j=1}^{n-1} \epsilon \Gamma\left(s_{n}-s_{j}+1\right) /\left(4 l\left|a_{j}\right|\right)\right)^{1 /\left(s_{n}-s_{n-1}\right)},\left(\epsilon \Gamma\left(s_{n}+1\right) /(4 M)\right)^{1 / s_{n}}\right\}$. For $0 \leq t_{1}<$ $t_{2} \leq 1, t_{2}-t_{1}<\delta$, we get

$$
\begin{aligned}
\left|u_{\epsilon}\left(t_{2}\right)-u_{\epsilon}\left(t_{1}\right)\right|= & \left|\sum_{j=1}^{n-1} I^{s_{n}-s_{j}} a_{j} u\left(t_{2}\right)-\sum_{j=1}^{n-1} I^{s_{n}-s_{j}} a_{j} u\left(t_{1}\right)+I^{s_{n}} f\left(t_{2}, u\left(t_{2}\right)\right)-I^{s_{n}} f\left(t_{1}, u\left(t_{1}\right)\right)\right| \\
= & \mid \sum_{j=1}^{n-1} \frac{a_{j}}{\Gamma\left(s_{n}-s_{j}\right)} \int_{0}^{t_{1}}\left[\left(t_{2}-s\right)^{s_{n}-s_{j}-1}-\left(t_{1}-s\right)^{s_{n}-s_{j}-1}\right] u(s) d s \\
& +\sum_{j=1}^{n-1} \frac{a_{j}}{\Gamma\left(s_{n}-s_{j}\right)} \int_{t_{1}}^{t_{2}}\left(t_{2}-s\right)^{s_{n}-s_{j}-1} u(s) d s+\frac{1}{\Gamma\left(s_{n}\right)} \int_{t_{1}}^{t_{2}}\left(t_{2}-s\right)^{s_{n}-1} f(s, u(s)) d s
\end{aligned}
$$


Abstract and Applied Analysis

$$
\begin{aligned}
& +\frac{1}{\Gamma\left(s_{n}\right)} \int_{0}^{t_{1}}\left[\left(t_{2}-s\right)^{s_{n}-1}-\left(t_{1}-s\right)^{s_{n}-1}\right] f(s, u(s)) d s \mid \\
\leq & \sum_{j=1}^{n-1} \frac{l\left|a_{j}\right|}{\Gamma\left(s_{n}-s_{j}\right)} \int_{0}^{t_{1}}\left[\left(t_{1}-s\right)^{s_{n}-s_{j}-1}-\left(t_{2}-s\right)^{s_{n}-s_{j}-1}\right] d s \\
& +\sum_{j=1}^{n-1} \frac{l\left|a_{j}\right|}{\Gamma\left(s_{n}-s_{j}\right)} \int_{t_{1}}^{t_{2}}\left(t_{2}-s\right)^{s_{n}-s_{j}-1} d s+\frac{M}{\Gamma\left(s_{n}\right)} \int_{t_{1}}^{t_{2}}\left(t_{2}-s\right)^{s_{n}-1} d s \\
& +\frac{M}{\Gamma\left(s_{n}\right)} \int_{0}^{t_{1}}\left[\left(t_{1}-s\right)^{s_{n}-1}-\left(t_{2}-s\right)^{s_{n}-1}\right] d s \\
= & \sum_{j=1}^{n-1} \frac{l\left|a_{j}\right|}{\Gamma\left(s_{n}-s_{j}+1\right)}\left(t_{1}^{s_{n}-s_{j}}-t_{2}^{s_{n}-s_{j}}\right)+\sum_{j=1}^{n-1} \frac{2 l\left|a_{j}\right|}{\Gamma\left(s_{n}-s_{j}+1\right)}\left(t_{2}-t_{1}\right)^{s_{n}-s_{j}} \\
& +\frac{M}{\Gamma\left(s_{n}+1\right)}\left(t_{1}^{s_{n}}-t_{2}^{s_{n}}\right)+\frac{2 M}{\Gamma\left(s_{n}+1\right)}\left(t_{2}-t_{1}\right)^{s_{n}} \\
\leq & \sum_{j=1}^{n-1} \frac{2 l\left|a_{j}\right|}{\Gamma\left(s_{n}-s_{j}+1\right)}\left(t_{2}-t_{1}\right)^{s_{n}-s_{n-1}}+\frac{2 M}{\Gamma\left(s_{n}+1\right)}\left(t_{2}-t_{1}\right)^{s_{n}} \\
\leq & \frac{\epsilon}{2}+\frac{\epsilon}{2}=\epsilon .
\end{aligned}
$$

Thus, $\left\{u_{\epsilon}(t)\right\}$ is equicontinuous on $[0,1]$.

Lemma 2.7 (see [16, Theorem 4.2]). Let $f:[0,1] \times \mathbb{R} \rightarrow \mathbb{R}$ be continuous and Lipschitz with respect to second variable with Lipschitz constant $L$. Let $a_{j}$ satisfy

$$
0<\frac{L}{\Gamma\left(s_{n}+1\right)}+\sum_{j=1}^{n-1} \frac{\left|a_{j}\right|}{\Gamma\left(s_{n}-s_{j}+1\right)}<1 .
$$

Then IVP (1.4) has a unique solution.

Lemma 2.8. Let $v, w \in C([0,1], \mathbb{R})$ be locally Hölder continuous with exponent $q<\lambda<1, f \in$ $C([0,1] \times \mathbb{R}, \mathbb{R})$ and

$$
L(D) w \geq f(t, w), \quad L(D) v \leq f(t, v), \quad 0<t \leq 1
$$

one of the nonstrict inequalities being strict. Then $v(0)<w(0)$ implies $v(t)<w(t), 0 \leq t \leq 1$.

Proof. Suppose that $v(t)<w(t), 0 \leq t \leq 1$ is not true. We suppose the inequality $L(D)>$ $f(t, w(t))$. Letting $m(t)=v(t)-w(t)$, there exists $0<t_{1} \leq 1$ such that $m(t) \leq 0,0 \leq t \leq t_{1}$, and 
$m\left(t_{1}\right)=0$. Then by Corollary 2.5, we can obtain $L(D) m\left(t_{1}\right) \geq 0$. From the conditions and the definition of $m(t)$, we have

$$
f\left(t_{1}, v\left(t_{1}\right)\right) \geq L(D) v\left(t_{1}\right) \geq L(D) w\left(t_{1}\right)>f\left(t_{1}, w\left(t_{1}\right)\right) .
$$

This is a contradiction to $v\left(t_{1}\right)=w\left(t_{1}\right)$. The proof is complete.

Lemma 2.9. Assume that the conditions of Lemma 2.8 hold with nonstrict inequalities (2.3) and (2.4). Furthermore, suppose that

$$
f(t, x)-f(t, y) \leq N(x-y), \quad \text { where } x \geq y, N>0
$$

Then $v(0) \leq w(0)$ implies $v(t) \leq w(t), 0 \leq t \leq 1$ provided $N<1 / \Gamma\left(1-s_{n}\right)-\sum_{j=1}^{n-1} a_{j} / \Gamma\left(1-s_{j}\right)$.

Proof. Let $w_{\epsilon}(t)=w(t)+\epsilon$. For small $\epsilon>0$, we have

$$
w_{\epsilon}(0)>w(0), \quad w_{\epsilon}(t)>w(t), \quad 0 \leq t \leq 1 .
$$

Then, from (2.11) and (2.12) we get

$$
\begin{aligned}
L(D) w_{\epsilon}(t) & =L(D) w(t)+L(D) \epsilon \\
& =f(t, w(t))+\epsilon\left[\frac{t^{-s_{n}}}{\Gamma\left(1-s_{n}\right)}-\sum_{j=1}^{n-1} \frac{a_{j} t^{-s_{j}}}{\Gamma\left(1-s_{j}\right)}\right] \\
& \geq f\left(t, w_{\epsilon}(t)\right)-N \epsilon+\epsilon\left[\frac{t^{-s_{n}}}{\Gamma\left(1-s_{n}\right)}-\sum_{j=1}^{n-1} \frac{a_{j} t^{-s_{j}}}{\Gamma\left(1-s_{j}\right)}\right] \\
& \geq f\left(t, w_{\epsilon}(t)\right)-N \epsilon+\epsilon\left[\frac{1}{\Gamma\left(1-s_{n}\right)}-\sum_{j=1}^{n-1} \frac{a_{j}}{\Gamma\left(1-s_{j}\right)}\right] \\
& >f\left(t, w_{\epsilon}(t)\right), \quad 0<t \leq 1 .
\end{aligned}
$$

Applying Lemma 2.8, we obtain $v(t)<w_{\epsilon}(t), 0 \leq t \leq 1$. By the arbitrariness of $\epsilon>0$, we can conclude that $v(t) \leq w(t)$. The proof is complete.

Corollary 2.10. The function $f(t, u)=\sigma(t) u$, where $\sigma(t) \leq N$, is admissible in Lemma 2.9 to yield $v(t) \leq 0$ on $0 \leq t \leq 1$.

\section{Main Results}

In this section, we establish the existence and uniqueness criteria of solutions for initial value problem (1.4). 
Theorem 3.1. Assume that $f \in C\left(R_{0}, \mathbb{R}\right)$, where $R_{0}=\{(t, u): 0 \leq t \leq 1,|u(t)| \leq b\}$ and $|f(t, u)| \leq$ $M$. Then IVP (1.4) possesses at least one solution $u(t)$ on $0 \leq t \leq \alpha$, where $\alpha=\min \{1,(b \Gamma(1+$ $\left.\left.\left.s_{n}\right) /(2 M)\right)^{1 / s_{n}},\left(\sum_{j=1}^{n-1} \Gamma\left(s_{n}-s_{j}+1\right) /\left(2\left|a_{j}\right|\right)\right)^{1 /\left(s_{n}-s_{n-1}\right)}\right\}$.

Proof. Let $u_{0}(t)$ be a continuous function on $[-\delta, 0], \delta>0$, such that $u_{0}(0)=0,\left|u_{0}(t)\right| \leq b$ and $\left|L(D) u_{0}(t)\right| \leq M$, where $D_{0-}^{s_{j}} u_{0}(t), j=1,2, \ldots, n-1$ are the continuous fractional derivatives. For $0<\epsilon \leq \delta$, we define the function $u_{\epsilon}(t)=u_{0}(t)$ on $[-\delta, 0]$ and

$$
u_{\epsilon}(t)=\frac{1}{\Gamma\left(s_{n}\right)} \int_{0}^{t}(t-s)^{s_{n}-1} f\left(s, u_{\epsilon}(s-\epsilon)\right) d s+\sum_{j=1}^{n-1} \frac{a_{j}}{\Gamma\left(s_{n}-s_{j}\right)} \int_{0}^{t}(t-s)^{s_{n}-s_{j}-1} u_{\epsilon}(s-\epsilon) d s
$$

on $\left[0, \alpha_{1}\right]$, where $\alpha_{1}=\min \{\epsilon, \alpha\}$. We observe that $D^{s_{j}} \mathcal{u}_{\epsilon}(t), j=1,2, \ldots, n$ exist for $t \in\left[0, \alpha_{1}\right]$ and

$$
\begin{aligned}
\left|u_{\epsilon}(t)\right| & \leq \frac{1}{\Gamma\left(s_{n}\right)} \int_{0}^{t}(t-s)^{s_{n}-1}\left|f\left(s, u_{\epsilon}(s-\epsilon)\right)\right| d s+\sum_{j=1}^{n-1} \frac{\left|a_{j}\right|}{\Gamma\left(s_{n}-s_{j}\right)} \int_{0}^{t}(t-s)^{s_{n}-s_{j}-1}\left|u_{\epsilon}(s-\epsilon)\right| d s \\
& \leq \frac{M}{\Gamma\left(s_{n}\right)} \int_{0}^{t}(t-s)^{s_{n}-1} d s+\sum_{j=1}^{n-1} \frac{b\left|a_{j}\right|}{\Gamma\left(s_{n}-s_{j}\right)} \int_{0}^{t}(t-s)^{s_{n}-s_{j}-1} d s \\
& =\frac{M}{\Gamma\left(s_{n}+1\right)} t^{s_{n}}+\sum_{j=1}^{n-1} \frac{b\left|a_{j}\right|}{\Gamma\left(s_{n}-s_{j}+1\right)} t^{s_{n}-s_{j}} \\
& \leq \frac{M}{\Gamma\left(s_{n}+1\right)} \alpha^{s_{n}}+\sum_{j=1}^{n-1} \frac{b\left|a_{j}\right|}{\Gamma\left(s_{n}-s_{j}+1\right)} \alpha^{s_{n}-s_{n-1}} \\
& \leq \frac{b}{2}+\frac{b}{2}=b .
\end{aligned}
$$

If $\alpha_{1}<\alpha$, we can employ (3.1) to extend $u_{\epsilon}(t)$ as a continuously fractional differentiable function on $\left[-\delta, \alpha_{2}\right], \alpha_{2}=\min \{\alpha, 2 \epsilon\}$ such that $u_{\epsilon}(t) \leq b$ holds. Continuing this process, we can define $u_{\epsilon}(t)$ over $[-\delta, \alpha]$ so that $u_{\epsilon}(t) \leq b$; it has a continuous fractional derivative and satisfies (3.1) on the same interval $[-\delta, \alpha]$. Furthermore, $\left|L(D) u_{\epsilon}(t)\right| \leq M$, since $\mid f\left(t, u_{\epsilon}(t-\right.$ $\epsilon)) \mid \leq M$ on $R_{0}$. Therefore, from Lemma 2.6, the family $\left\{u_{\epsilon}(t)\right\}$ is an equicontinuous and uniformly bounded function. An application of Ascoli-Arzela Theorem shows the existence of a sequence $\left\{\epsilon_{n}\right\}$ such that $\epsilon_{1}>\epsilon_{2}>\cdots>\epsilon_{n} \rightarrow 0$ as $n \rightarrow \infty$, and $u(t)=\lim _{n \rightarrow \infty} u_{\epsilon_{n}}(t)$ exists uniformly on $[-\delta, \alpha]$. Due to $f$ being uniformly continuous, we can obtain $f\left(t, u_{\epsilon_{n}}(t-\right.$ $\left.\epsilon_{n}\right)$ ) which uniformly tends to $f(t, u(t))$, and $u_{\epsilon_{n}}\left(t-\epsilon_{n}\right)$ uniformly tends to $u(t)$ as $n \rightarrow \infty$. Therefore, term by term, integration of (3.1) with $\epsilon=\epsilon_{n}, \alpha_{1}=\alpha$ yields

$$
u(t)=\frac{1}{\Gamma\left(s_{n}\right)} \int_{0}^{t}(t-s)^{s_{n}-1} f(s, u(s)) d s+\sum_{j=1}^{n-1} \frac{a_{j}}{\Gamma\left(s_{n}-s_{j}\right)} \int_{0}^{t}(t-s)^{s_{n}-s_{j}-1} u(s) d s .
$$

This proves that $u(t)$ is a solution of IVP (1.4) and the proof is complete. 
Theorem 3.2. Let $v, w \in C([0,1], \mathbb{R})$ be lower and upper solutions of the IVP (1.4) which are locally Hölder continuous with exponent $s_{n}<\lambda<1$ such that $v(t) \leq w(t), t \in[0,1]$ and $f \in C(\Omega, \mathbb{R})$, where $\Omega=\{(t, u): v(t) \leq u(t) \leq w(t), t \in[0,1]\}$. Furthermore, suppose that

$$
\left(\sum_{j=1}^{n-1} \frac{\Gamma\left(s_{n}-s_{j}+1\right)}{2\left|a_{j}\right|}\right)^{1 /\left(s_{n}-s_{n-1}\right)} \geq 1
$$

Then there exists a solution $u(t)$ of IVP (1.4) satisfying $v(t) \leq u(t) \leq w(t)$ on $[0,1]$.

Proof. For the need of proof, we define function $p(t, u):[0,1] \times \mathbb{R} \rightarrow \mathbb{R}$ as

$$
p(t, u)=\max \{v(t), \min \{u, w(t)\}\}
$$

Therefore, $f(t, p(t, u))$ defines a continuous extension of $f$ to $[0,1] \times R$ which is also bounded because $f$ is bounded on $\Omega$. Then by Theorems 3.1 and 3.2, we can obtain that the initial value problem

$$
\begin{gathered}
L(D) u=f(t, p(t, u)), \quad t \in(0,1], \\
u(0)=0,
\end{gathered}
$$

has a solution on $[0,1]$.

Clearly, from the definition of function $p(t, u)$, we know that if IVP (3.6) exits a solution $u(t)$ satisfying $v(t) \leq u(t) \leq w(t)$ on $[0,1]$, then $u(t)$ is also a solution of IVP (1.4). In the following, we will prove that the solution $u(t)$ of IVP (3.6) satisfies $v(t) \leq u(t) \leq w(t)$ on $[0,1]$.

For any $\epsilon>0$, we consider

$$
w_{\epsilon}(t)=w(t)+\epsilon, \quad v_{\epsilon}(t)=v(t)-\epsilon .
$$

Then, we get

$$
w_{\epsilon}(0)=w(0)+\epsilon, \quad v_{\epsilon}(0)=v(0)-\epsilon .
$$

Therefore, it follows that $v_{\epsilon}(0)<u(0)<w_{\epsilon}(0)$. Next, we will show that $v_{\epsilon}(t)<u(t)<w_{\epsilon}(t)$, $t \in[0,1]$. Suppose that it is not true. Then there exists $t_{1} \in(0,1]$ such that

$$
u\left(t_{1}\right)=w_{\epsilon}\left(t_{1}\right), \quad v_{\epsilon}(t)<u(t)<w_{\epsilon}(t), \quad 0 \leq t<t_{1} .
$$


Therefore, $u\left(t_{1}\right)>w\left(t_{1}\right), p\left(t_{1}, u\left(t_{1}\right)\right)=w\left(t_{1}\right)$ and $v\left(t_{1}\right) \leq p\left(t_{1}, u\left(t_{1}\right)\right) \leq w\left(t_{1}\right)$. Letting $m(t)=$ $u(t)-w_{\epsilon}(t)$, we have $m\left(t_{1}\right)=0$ and $m(t) \leq 0,0 \leq t \leq t_{1}$. Then from Corollary 2.5, we can obtain $L(D) m\left(t_{1}\right) \geq 0$ and

$$
\begin{aligned}
f\left(t_{1}, w\left(t_{1}\right)\right) & =f\left(t_{1}, p\left(t_{1}, w\left(t_{1}\right)\right)\right) \\
& =L(D) u\left(t_{1}\right) \geq L(D) w_{\epsilon}\left(t_{1}\right) \\
& =L(D) w\left(t_{1}\right)+L(D) \epsilon\left(t_{1}\right) \\
& =L(D) w\left(t_{1}\right)+\epsilon\left[\frac{t_{1}^{-s_{n}}}{\Gamma\left(1-s_{n}\right)}-\sum_{j=1}^{n-1} \frac{a_{j} t_{1}^{-S_{j}}}{\Gamma\left(1-s_{j}\right)}\right] \\
& >L(D) w\left(t_{1}\right)=f\left(t_{1}, w\left(t_{1}\right)\right),
\end{aligned}
$$

which is a contradiction. The other case $v_{\epsilon}(t)<u(t)$ can be proved similarly.

Hence, we get $v_{\epsilon}(t)<u(t)<w_{\epsilon}(t)$ on $[0,1]$. Letting $\epsilon \rightarrow 0$, we obtain $v(t) \leq u(t) \leq$ $w(t)$ on $[0,1]$. The proof is complete.

Now, we will give the existence of maximal and minimal solutions of initial value problem (1.4).

Theorem 3.3. Let $f \in C([0,1] \times \mathbb{R}, \mathbb{R}), v_{0}, w_{0}$ be lower and upper solutions of (1.4) such that $v_{0} \leq w_{0}$ on $[0,1]$. Furthermore, suppose that

$$
f(t, x)-f(t, y) \geq-N(x-y), \quad \text { for } \mathrm{v}_{0} \leq \mathrm{y} \leq \mathrm{x} \leq \mathrm{w}_{0}, \mathrm{~N} \geq 0,
$$

and $a_{j}$ satisfy

$$
0<\frac{N}{\Gamma\left(s_{n}+1\right)}+\sum_{j=1}^{n-1} \frac{\left|a_{j}\right|}{\Gamma\left(s_{n}-s_{j}+1\right)}<1 .
$$

Then there exist monotone sequences $\left\{v_{n}\right\}$ and $\left\{w_{n}\right\}$ such that $v_{n} \rightarrow \rho, w_{n} \rightarrow r$ as $n \rightarrow \infty$ uniformly on $[0,1]$, where $\rho$ and $r$ are minimal and maximal solutions of IVP $(1.4)$, respectively.

Proof. For any $\eta \in C([0,1], \mathbb{R})$ satisfying $v_{0} \leq \eta \leq w_{0}$, we consider the following linear fractional differential equation:

$$
\begin{gathered}
L(D) u=f(t, \eta)-N(u-\eta), \quad t \in(0,1], \\
u(0)=0 .
\end{gathered}
$$

Obviously, the right hand side of (3.13) satisfies the Lipschitz condition. From (3.11) and Lemma 2.7, it is clear that for every $\eta$, there exists a unique solution $u$ of $(3.13)$ on $[0,1]$. 
Define the operator $T$ by $T \eta=u$ and use it to construct the sequences $\left\{v_{n}\right\},\left\{w_{n}\right\}$. We need to prove the following propositions hold:

(i) $v_{0} \leq T v_{0}, w_{0} \geq T w_{0}$ ；

(ii) $T$ is a monotone operator on the segment

$$
\left\langle v_{0}, w_{0}\right\rangle=\left\{u \in C([0,1], \mathbb{R}): v_{0} \leq u \leq w_{0}\right\}
$$

To prove (i), let $T v_{0}=v_{1}$, where $v_{1}$ is the unique solution of (3.13) with $\eta=v_{0}$. Letting $p=v_{0}-v_{1}$, we have

$$
\begin{gathered}
L(D) p=L(D) v_{0}-L(D) v_{1} \leq f\left(t, v_{0}\right)-\left[f\left(t, v_{0}\right)-N\left(v_{1}-v_{0}\right)\right]=-N p, \\
p(0)=v_{0}(0)-v_{1}(0) \leq 0 .
\end{gathered}
$$

By Corollary 2.10, we can obtain that $p(t) \leq 0$ on $[0,1]$, that is, $v_{0} \leq T v_{0}$.

Similarly, we can get $w_{0} \geq T w_{0}$.

To prove (ii), let $\eta_{1}, \eta_{2} \in\left\langle v_{0}, w_{0}\right\rangle$ such that $\eta_{1} \leq \eta_{2}$. Assume that $u_{1}=T \eta_{1}$ and $u_{2}=T \eta_{2}$. Setting $p=u_{1}-u_{2}$, then using the condition (3.11), we have

$$
\begin{gathered}
L(D) p=L(D) u_{1}-L(D) u_{2}=f\left(t, \eta_{1}\right)-N\left(u_{1}-\eta_{1}\right)-\left[f\left(t, \eta_{2}\right)-N\left(u_{2}-\eta_{2}\right)\right] \\
\leq-N\left(\eta_{1}-\eta_{2}\right)-N\left(u_{1}-\eta_{1}\right)+N\left(u_{2}-\eta_{2}\right)=-N p \\
p(0)=u_{1}(0)-u_{2}(0)=0 .
\end{gathered}
$$

From Corollary 2.10, we can obtain that $p(t) \leq 0$ on $[0,1]$, which implies $T \eta_{1} \leq T \eta_{2}$. And (ii) is proved.

Therefore, we can define the sequences $v_{n}=T v_{n-1}, w_{n}=T w_{n-1}$. From the previous discussion, we can get

$$
v_{0} \leq v_{1} \leq \cdots \leq v_{n} \leq w_{n} \leq \cdots \leq w_{1} \leq w_{0} \quad \text { on }[0,1]
$$

Clearly, the sequences $\left\{v_{n}\right\},\left\{w_{n}\right\}$ are uniformly bounded on $[0,1]$. From (3.13), we have $\left|L(D) v_{n}\right|,\left|L(D) w_{n}\right|$ which are also uniformly bounded. By Lemma 2.6, we know that $\left\{v_{n}\right\},\left\{w_{n}\right\}$ are equicontinuous on $[0,1]$. Then applying Ascoli-Arzela Theorem, there exist subsequences $\left\{v_{n_{k}}\right\},\left\{w_{n_{k}}\right\}$ that converge uniformly on $[0,1]$. From (3.17), we can see that the entire sequences $\left\{v_{n}\right\},\left\{w_{n}\right\}$ converge uniformly and monotonically to $\rho, r$, respectively, as $n \rightarrow \infty$. It is now easy to show that $\rho, r$ are solutions of IVP (1.4) by the corresponding Volterra fractional integral equation for (3.13).

In the following, we will prove that $\rho$ and $r$ are the minimal and maximal solutions of IVP (1.4), respectively. We need to show that if $u$ is any solution of IVP (1.4) satisfying $v_{0} \leq u \leq w_{0}$ on $[0,1]$, then we have $v_{0} \leq \rho \leq u \leq r \leq w_{0}$ on $[0,1]$. 
We assume that for some $n, v_{n} \leq u \leq w_{n}$ on $[0,1]$ and letting $p=v_{n+1}-u$, we have

$$
\begin{gathered}
L(D) p=L(D) v_{n+1}-L(D) u=f\left(t, v_{n}\right)-N\left(v_{n+1}-v_{n}\right)-f(t, u) \\
\leq-N\left(v_{n}-u\right)-N\left(v_{n+1}-v_{n}\right)=-N p \\
p(0)=v_{n+1}(0)-u(0)=0
\end{gathered}
$$

which implies $v_{n+1} \leq u$. Similarly, we have $u \leq w_{n+1}$ on $[0,1]$. Since $v_{0} \leq u \leq w_{0}$ on $[0,1]$, this proves $v_{n} \leq u \leq w_{n}$ for all $n$ by induction. Letting $n \rightarrow \infty$, we conclude that $\rho \leq u \leq r$ on $[0,1]$ and the proof is complete.

Theorem 3.4. Suppose that the conditions of Theorem 3.3 hold. In addition, we assume

$$
f(t, x)-f(t, y) \leq N(x-y), \quad v_{0} \leq y \leq x \leq w_{0}, \quad N>0 .
$$

Then $\rho=r=u$ is the unique solution of IVP (1.4) provided $N<1 / \Gamma\left(1-s_{n}\right)-\sum_{j=1}^{n-1} a_{j} / \Gamma\left(1-s_{j}\right)$.

Proof. We have proved $\rho \leq r$ in Theorem 3.3, so we just need to prove $\rho \geq r$. Letting $p=r-\rho$, we get

$$
\begin{gathered}
L(D) p=f(t, r)-f(t, \rho) \leq N p, \\
p(0)=r(0)-\rho(0)=0 .
\end{gathered}
$$

From Corollary 2.10, we obtain $p \leq 0$ on $[0,1]$, which implies $\rho \geq r$. Hence, $\rho=r=u$ is the unique solution of IVP (1.4).

\section{Examples}

In this paper, we will present an example to illustrate the main results.

Example 4.1. Consider the initial value problem of fractional differential equation

$$
\begin{gathered}
D^{0.8} u+0.4 D^{0.6} u=\frac{u^{2} t^{0.2}}{10 \Gamma(0.2)}-\frac{u t^{0.4}}{2 \Gamma(0.4)}, \quad t \in(0,1] \\
u(0)=0 .
\end{gathered}
$$

Choose $w=5, v=-5$; then we can obtain

$$
\begin{gathered}
D^{0.8} w+0.4 D^{0.6} w \geq \frac{w^{2} t^{0.2}}{10 \Gamma(0.2)}-\frac{w t^{0.4}}{2 \Gamma(0.4)}, \\
D^{0.8} v+0.4 D^{0.6} v \leq \frac{v^{2} t^{0.2}}{10 \Gamma(0.2)}-\frac{v t^{0.4}}{2 \Gamma(0.4)} .
\end{gathered}
$$


That is, $v$ and $w$ are the lower and upper solutions of initial value problem (4.1). Furthermore, $v$ and $w$ are locally continuous with exponent $1>\lambda>0.8$.

Since

$$
\left(\frac{\Gamma(0.8-0.6+1)}{2|-0.4|}\right)^{1 /(0.8-0.6)}=1.9914>1
$$

then by Theorem 3.2, there exists a solution $u(t)$ of initial value problem (4.1) satisfying $-5 \leq$ $u(t) \leq 5$.

Next, we will prove the existence of maximal and minimal solutions for initial value problem (4.1) by using Theorem 3.3.

Let $v_{0}=-5$ and $w_{0}=5$ be lower and upper solutions of (4.1). Furthermore, for any $-5 \leq y \leq x \leq 5$, we have

$$
\begin{aligned}
f(t, x)-f(t, y) & =\frac{x^{2} t^{0.2}}{10 \Gamma(0.2)}-\frac{x t^{0.4}}{2 \Gamma(0.4)}-\frac{y^{2} t^{0.2}}{10 \Gamma(0.2)}+\frac{y t^{0.4}}{2 \Gamma(0.4)} \\
& =\frac{t^{0.2}}{10 \Gamma(0.2)}(x-y)(x+y)-\frac{t^{0.4}}{2 \Gamma(0.4)}(x-y) \\
& \geq-\frac{1}{2 \Gamma(0.4)}(x-y) .
\end{aligned}
$$

Then let $N=1 / 2 \Gamma(0.4) \approx 0.2254$. We get

$$
0<\frac{N}{\Gamma(0.8+1)}+\frac{|-0.4|}{\Gamma(0.8-0.6+1)} \approx 0.6777<1
$$

Thus, from Theorem 3.3, there exist monotone sequences $\left\{v_{n}\right\}$ and $\left\{w_{n}\right\}$ such that $v_{n} \rightarrow \rho$, $w_{n} \rightarrow r$ as $n \rightarrow \infty$ uniformly on [0,1], where $\rho$ and $r$ are minimal and maximal solutions of initial value problem (4.1), respectively.

In addition,

$$
\begin{aligned}
f(t, x)-f(t, y) & =\frac{x^{2} t^{0.2}}{10 \Gamma(0.2)}-\frac{x t^{0.4}}{2 \Gamma(0.4)}-\frac{y^{2} t^{0.2}}{10 \Gamma(0.2)}+\frac{y t^{0.4}}{2 \Gamma(0.4)} \\
& =\frac{t^{0.2}}{10 \Gamma(0.2)}(x-y)(x+y)-\frac{t^{0.4}}{2 \Gamma(0.4)}(x-y) \\
& \leq \frac{10}{10 \Gamma(0.2)}(x-y) \\
& \leq N(x-y), \\
0<N & <\frac{1}{\Gamma(1-0.8)}-\frac{-0.4}{\Gamma(1-0.6)} \approx 0.3982 .
\end{aligned}
$$

Hence, by Theorem 3.4, initial value problem (4.1) has a unique solution. 


\section{Conclusion}

In this paper, we considered the initial value problem of nonlinear fractional differential equation

$$
\begin{gathered}
L(D) u=f(t, u), \quad t \in(0,1], \\
u(0)=0 .
\end{gathered}
$$

The basic theory of differential equation, the method of upper and lower solutions, and monotone iterative technique have been applied for the existence and uniqueness of solutions of the initial value problem. And several results were obtained. Besides, we studied the existence of minimal and maximal solutions. In Section 4, we also give an example to illustrate our results.

\section{Acknowledgments}

This research is supported by the Natural Science Foundation of China (11071143, 60904024, 61174217), Natural Science Outstanding Youth Foundation of Shandong Province (JQ201119), supported by Shandong Provincial Natural Science Foundation (ZR2010AL002, ZR2009AL003), and supported by Natural Science Foundation of Educational Department of Shandong Province (J11LA01).

\section{References}

[1] A. A. Kilbas, H. M. Srivastava, and J. J. Trujillo, Theory and Applications of Fractional Differential Equations, vol. 204, Elsevier Science, Amsterdam, The Netherlands, 2006.

[2] K. S. Miller and B. Ross, An Introduction to the Fractional Calculus and Differential Equations, John Wiley \& Sons, New York, NY, USA, 1993.

[3] I. Podlubny, Fractional Differential Equations, vol. 198, Academic Press, San Diego, Ca, USA, 1999.

[4] D. Baleanu, K. Diethelm, E. Scalas, and J. J. Trujillo, Fractional Calculus Models and Numerical Methods, vol. 3 of Series on Complexity, Nonlinearity and Chaos, World Scientific, Hackensack, NJ, USA, 2012.

[5] A. E. M. El-Mesiry, A. M. A. El-Sayed, and H. A. A. El-Saka, "Numerical methods for multi-term fractional (arbitrary) orders differential equations," Applied Mathematics and Computation, vol. 160, no. 3, pp. 683-699, 2005.

[6] I. Hashim, O. Abdulaziz, and S. Momani, "Homotopy analysis method for fractional IVPs," Communications in Nonlinear Science and Numerical Simulation, vol. 14, no. 3, pp. 674-684, 2009.

[7] Y.-K. Chang, V. Kavitha, and M. Mallika Arjunan, "Existence and uniqueness of mild solutions to a semilinear integrodifferential equation of fractional order," Nonlinear Analysis A, vol. 71, no. 11, pp. 5551-5559, 2009.

[8] A. Chen, F. Chen, and S. Deng, "On almost automorphic mild solutions for fractional semilinear initial value problems," Computers \& Mathematics with Applications, vol. 59, no. 3, pp. 1318-1325, 2010.

[9] D. Delbosco and L. Rodino, "Existence and uniqueness for a nonlinear fractional differential equation," Journal of Mathematical Analysis and Applications, vol. 204, no. 2, pp. 609-625, 1996.

[10] V. Lakshmikantham and A. S. Vatsala, "Basic theory of fractional differential equations," Nonlinear Analysis A, vol. 69, no. 8, pp. 2677-2682, 2008.

[11] V. Lakshmikantham and A. S. Vatsala, "Theory of fractional differential inequalities and applications," Communications in Applied Analysis, vol. 11, no. 3-4, pp. 395-402, 2007.

[12] V. Lakshmikantham and A. S. Vatsala, "General uniqueness and monotone iterative technique for fractional differential equations," Applied Mathematics Letters, vol. 21, no. 8, pp. 828-834, 2008.

[13] J. D. Ramírez and A. S. Vatsala, "Monotone iterative technique for fractional differential equations with periodic boundary conditions," Opuscula Mathematica, vol. 29, no. 3, pp. 289-304, 2009. 
[14] F. A. McRae, "Monotone iterative technique and existence results for fractional differential equations," Nonlinear Analysis A, vol. 71, no. 12, pp. 6093-6096, 2009.

[15] S. Zhang, "Monotone iterative method for initial value problem involving Riemann-Liouville fractional derivatives," Nonlinear Analysis A, vol. 71, no. 5-6, pp. 2087-2093, 2009.

[16] A. Babakhani and V. Daftardar-Gejii, "Existence of positive solutions of nonlinear fractional differential equations," Journal of Mathematical Analysis and Applications, vol. 278, no. 2, pp. 434-442, 2003.

[17] S. Q. Zhang, "Existence of positive solution for a singular initial value problem for a fractional differential equation," Acta Mathematica Sinica, vol. 50, no. 4, pp. 813-822, 2007.

[18] V. S. Erturk, S. Momani, and Z. Odibat, "Application of generalized differential transform method to multi-order fractional differential equations," Communications in Nonlinear Science and Numerical Simulation, vol. 13, no. 8, pp. 1642-1654, 2008.

[19] A. Arikoglu and I. Ozkol, "Comments on "Application of generalized differential transform method to multi-order fractional differential equations", Vedat Suat Erturk, Shaher Momani, Zaid Odibat [Commun Nonlinear Sci Numer Simul 2008;13:1642-54]," Communications in Nonlinear Science and Numerical Simulation, vol. 13, no. 8, pp. 1737-1740, 2008.

[20] D. Băleanu, O. G. Mustafa, and R. P. Agarwal, "On the solution set for a class of sequential fractional differential equations," Journal of Physics A, vol. 43, no. 38, article 385209, p. 7, 2010.

[21] S. Sun, Y. Zhao, Z. Han, and M. Xu, "Uniqueness of positive solutions for boundary value problems of singular fractional differential equations," Inverse Problems in Science and Engineering, vol. 20, no. 3, pp. 299-309, 2012.

[22] Y. Zhao, S. Sun, Z. Han, and M. Zhang, "Positive solutions for boundary value problems of nonlinear fractional differential equations," Applied Mathematics and Computation, vol. 217, no. 16, pp. 6950-6958, 2011.

[23] Y. Zhao, S. Sun, Z. Han, and Q. Li, "Positive solutions to boundary value problems of nonlinear fractional differential equations," Abstract and Applied Analysis, vol. 2011, Article ID 390543, 16 pages, 2011.

[24] W. Feng, S. Sun, Z. Han, and Y. Zhao, "Existence of solutions for a singular system of nonlinear fractional differential equations," Computers $\mathcal{E}$ Mathematics with Applications, vol. 62, no. 3, pp. 1370 1378, 2011.

[25] S. Zhang, "The existence of a positive solution for a nonlinear fractional differential equation," Journal of Mathematical Analysis and Applications, vol. 252, no. 2, pp. 804-812, 2000.

[26] Z. Bai and H. Lü, "Positive solutions for boundary value problem of nonlinear fractional differential equation," Journal of Mathematical Analysis and Applications, vol. 311, no. 2, pp. 495-505, 2005.

[27] Y. Zhao, S. Sun, Z. Han, and Q. Li, "The existence of multiple positive solutions for boundary value problems of nonlinear fractional differential equations," Communications in Nonlinear Science and Numerical Simulation, vol. 16, no. 4, pp. 2086-2097, 2011.

[28] S. Sun, Q. Li, and Y. Li, "Existence and uniqueness of solutions for a coupled system of multi-term nonlinear fractional differential equations," Computers and Mathematics with Applications. In press.

[29] G. Wang, R. P. Agarwal, and A. Cabada, "Existence results and the monotone iterative technique for systems of nonlinear fractional differential equations," Applied Mathematics Letters, vol. 25, no. 6, pp. 1019-1024, 2012.

[30] G. Wang, "Monotone iterative technique for boundary value problems of a nonlinear fractional differential equation with deviating arguments," Journal of Computational and Applied Mathematics, vol. 236, no. 9, pp. 2425-2430, 2012. 


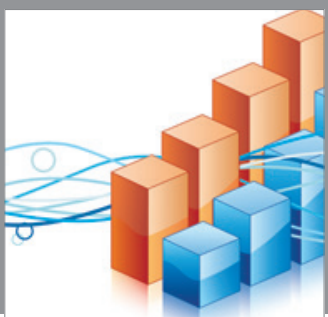

Advances in

Operations Research

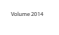

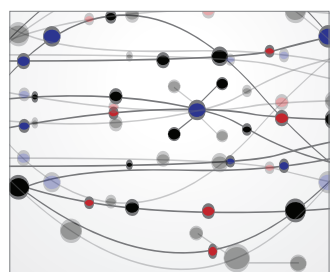

\section{The Scientific} World Journal
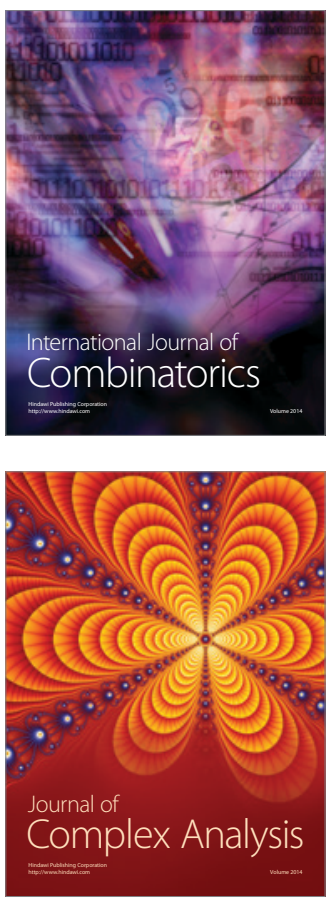

International Journal of

Mathematics and

Mathematical

Sciences
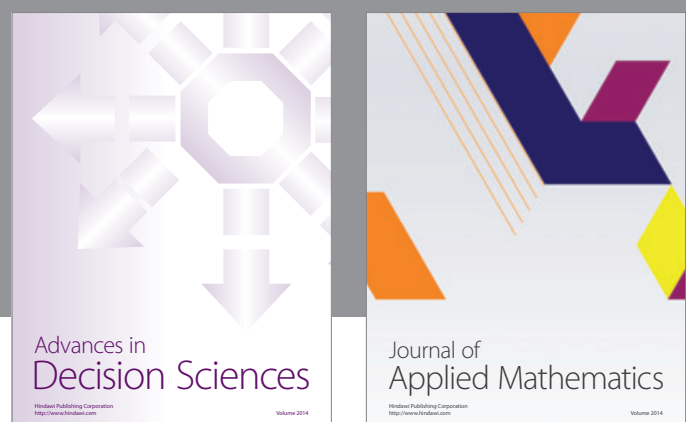

Journal of

Applied Mathematics
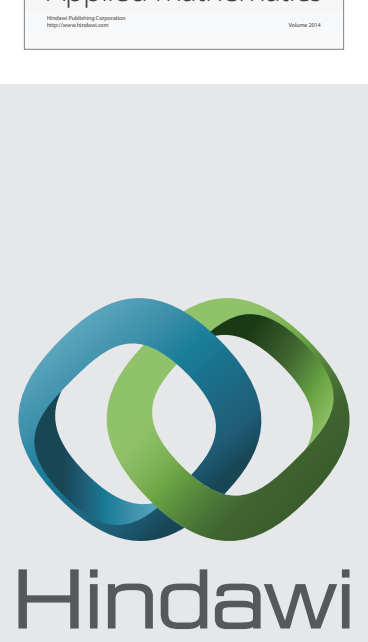

Submit your manuscripts at http://www.hindawi.com
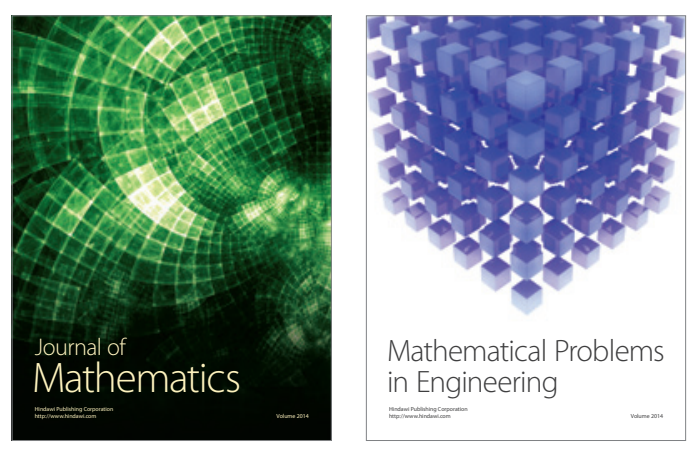

Mathematical Problems in Engineering
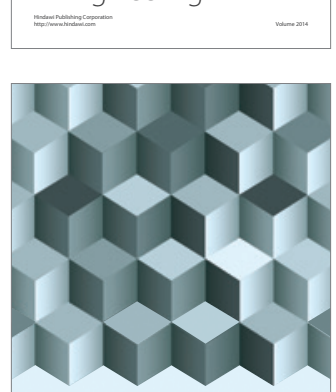

Journal of

Function Spaces
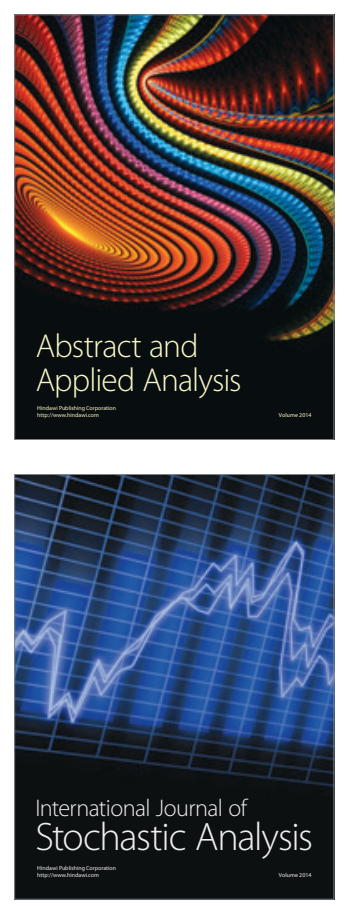

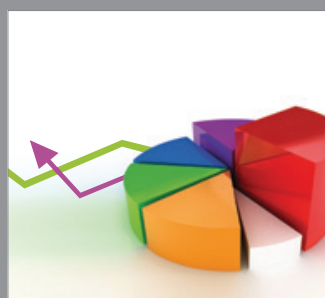

ournal of

Probability and Statistics

Promensencen
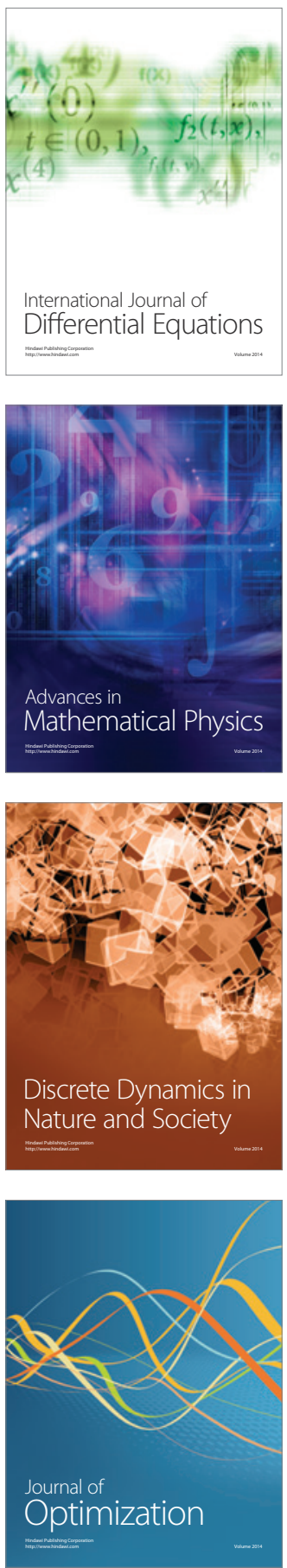